\title{
PROPRIEDADES DAS SÉRIES TEMPORAIS DE LUCROS TRIMESTRAIS DAS EMPRESAS BRASILEIRAS
}

\section{BRAZILIAN COMPANIES QUARTERLY PROFIT TIME SERIES PROPERTIES}

\author{
ANTONIO LOPO MARTINEZ \\ Doutor em Controladoria e Contabilidade pela \\ Universidade de São Paulo \\ Professor Adjunto da Faculdade de Ciências Contábeis da \\ Universidade Federal da Bahia \\ E-mail: antoniolodo@terra.com.br \\ NEWTON CARNEIRO AFFONSO DA COSTA JR \\ Doutor em Administração de Empresas pela \\ Fundação Getúlio Vargas/SP \\ Professor do Centro Sócio-Econômico da \\ Universidade Federal de Santa Catarina \\ E-mail: newton@cse.ufsc.br
}

\author{
CÉSAR MEDEIROS CUPERTINO \\ Mestre em Ciências Contábeis pela \\ Universidade de Brasília \\ Professor da Sociedade Educacional de Santa Catarina \\ Fundação Getúlio Vargas \\ E-mail: cupertino.cmc@gmail.com \\ REINALDO ALMEIDA COELHO \\ Mestre em Economia pela \\ Universidade Federal de Santa Catarina \\ Professor Colaborador da Escola Superior de \\ Administração e Gerência da \\ Universidade do Estado de Santa Catarina \\ E-mail: reinaldo@agce.com.br
}

\section{Resumo}

0 artigo analisa as propriedades das séries temporais de lucros trimestrais de empresas brasileiras, verificando a presença ou não de independência estatística nessas séries. Como variável síntese do desempenho da empresa, o lucro é objeto intenso de análise (por analistas de mercado, investidores, órgãos governamentais de fiscalização etc), fato que ressalta a relevância da pesquisa em relação à aleatoriedade do comportamento de sua série temporal. Os dados foram obtidos no banco de dados da Economatica para o período compreendido entre o primeiro trimestre de 1995 ao último trimestre de 2006. As variáveis utilizadas no estudo foram o Lucro Operacional e o Lucro Líquido, ambas submetidas aos seguintes testes: (i) mudanças médias; (ii) testes de carreiras (runs tests), (iii) diferenças sucessivas e (iv) correlação serial. Os resultados encontrados corroboram a evidência internacional sobre lucros trimestrais de que estes apresentam certa dependência temporal. Verificou-se que existe uma relação negativa entre variações sucessivas de lucros trimestrais, ou seja, empresas que aumentaram seus lucros acima da média num determinado período tiveram uma tendência a variar menos que a média nos períodos subseqüentes.

Palavras-chave: séries temporais; lucro operacional; lucro líquido; lucros trimestrais.

\section{Abstract}

This article analyzes the Brazilian companies quarterly profit time series properties, verifying whether there is or not the presence of statistics independence in these series. Being a variable summary of the company performance, the profit is strongly analyzed (by market analysts, investors, government tax department etc), which shows the relevance of research when compared to the random behavior of its time series. The data were obtained from the data base of Economatica for a period between the first quarterly of 1995 and the last one of 2006. The variables used in this study were: the operational profit and the net profit, both subjected to the tests: (i) average changes; (ii) run tests; (iii) successive differences and (iv) serial correlation. The results found show certain time dependency according previous international evidence. It was also verified that there was a negative relation between the quarterly profit successive variations which means the companies that had their profit increased above average during a certain time, tended to vary less than the others in subsequent periods.

Key words: time series, operating profit, net income, quarterly income. 


\section{INTRODUÇÃO}

Como medida síntese do desempenho da empresa, o lucro é utilizado por uma vasta faixa de usuários. Dechow (1992) relata seu emprego no cálculo da bonificação paga aos executivos de grandes corporações dos Estados Unidos da América. Outros exemplos incluem a monitoração da capacidade das empresas em liquidar as dívidas assumidas (SMITH \& WARNER, 1979); inclusão como variável em modelos de avaliação de empresas (OHLSON, 1995; FERNÁNDEZ, 2001); verificação do comportamento do preço de ações (DONNELLY, 2002); tratamento de questões contábeis (gerenciamento de lucros, por exemplo) etc.

A diversidade do uso dos lucros reportados suscita uma necessária digressão sobre a sua forma de mensuração. Entre as definições e maneiras de cálculo existentes, aquela que se apresenta de forma mais objetiva é dada pela contabilidade. Os princípios contábeis dão o balizamento elementar para os propósitos de uniformidade e comparabilidade ${ }^{1}$. Contudo, a contabilidade permite algumas ações discricionárias, admitindo critérios diferenciados, porém, igualmente aceitos, para registrar um determinado evento. Exemplos incluem a avaliação de estoques e depreciação de ativos tangíveis. Na visão de Strickland (1981), os administradores usam essa tolerância como fator de gerenciamento dos lucros a serem reportados, como, por exemplo, a prática bem conhecida de suavização (smoothing).

A análise do comportamento das séries temporais de lucros assume papel proeminente na identificação das características de interesse (tendências, "suavização" etc) para contadores, financistas, investidores e demais usuários. Nesse sentido, grande parte da pesquisa empírica confirma a independência entre as mudanças observadas nas séries de lucros (HAMMAN et al., 2002; BALL \& BROWN, 1969; BALL \& WATTS, 1972; LITTLE, 1962). Por outro lado, há diferentes teorias (como as "grafistas"2) que defendem que as séries passadas ão ricas em informações a respeito do comportamento futuro dos lucros das empresas. Em termos gerais, essa última linha dita que o histórico de lucros irá se repetir em um padrão de valores já observados e, portanto, o comportamento das séries futuras será recorrente.

A linha que defende a independência das séries de lucros é conhecida na literatura especializada como teoria Random Walk. Ela estabelece que o caminho futuro dos lucros não é mais previsível do que o caminho de números aleatórios acumulados. Em termos estatísticos, a teoria dita que as mudanças sucessivas em lucros são independentes, tal como se observa em variáveis distribuídas aleatoriamente. A implicação, então, é a de que a série não teria memória e o passado não poderia ser utilizado para predizer o futuro em nenhuma forma significativa.

O fato de que as mudanças nos lucros apresentam um comportamento temporal de distribuição aleatória apresenta uma série de implicações para investidores. A primeira delas é a de que, se a premissa for válida, as mudanças no lucro futuro de empresas, individualmente consideradas, não podem ser previstas a partir das variações já observadas em uma série histórica. Se as mudanças não podem ser previstas, as taxas de crescimento da variável também não podem ser estimadas. Hamman et al. (2002)

\footnotetext{
${ }^{1}$ Discussão detalhada sobre os princípios contábeis pode ser encontrada em Paton \& Littleton (1940) e em diversos livros-textos como, por exemplo, FIPECAFI (2003). No Brasil, os princípios fundamentais de contabilidade são regulamentados atualmente pela Resolução 750/93 do Conselho Federal de Contabilidade (1993).

${ }^{2}$ Chartist Theories, como é mais conhecida.
} 
extrapolam tais observações e ressaltam que, se a proposição é correta, todas ações devem ter a mesma taxa de crescimento esperada.

0 presente artigo analisa o comportamento da série de lucros de empresas brasileiras, verificando se há presença de independência estatística dos dados e, assim, confirmando (negando) a aplicação da teoria do Random Walk para a série de lucros. Optou-se por não utilizar um único teste, a fim de garantir uma maior robustez das constatações, assim como foram utilizadas duas medidas de lucros, mensuradas trimestralmente: Lucro Operacional (LO) e Lucro Líquido (LL).

As observações selecionadas para o Lucro Operacional e Lucro Líquido foram submetidas aos seguintes testes: (i) mudanças médias; (ii) teste de carreiras (runs test); (iii) diferenças sucessivas; (iv) correlação serial. 0 enfoque adotado em cada procedimento é explicado na seção 3.

Esta pesquisa é particularmente importante para se identificar o conteúdo informativo e preditivo do lucro trimestral no Brasil. Um dos objetivos gerais da contabilidade é proporcionar informações que possam ser usadas para prever eventos futuros. A compreensão do grau de previsibilidade dos lucros é importante para modelos de avaliação de empresas que, diretamente ou indiretamente, utilizem previsões de lucros. Outra forte motivação para a pesquisa é o fato de que estudos mais recentes têm demonstrado (HONG \& STEIN 1999), de modo contrário à hipótese de eficiência do mercado, que os retornos em ações são previsíveis e estão associados às propriedades dos lucros, o que cria uma demanda por pesquisa nas relacionadas a previsões de lucros.

0 restante do estudo é organizado como segue: a seção 2 descreve a evidência empírica anterior, a seção 3 estabelece a metodologia e os dados empregados, a seção 4 analisa os resultados e a seção 5 conclui o trabalho.

\section{EVIDÊNCIA ANTERIOR}

A lucratividade das empresas tem sido objeto de intensa pesquisa nas últimas quatro décadas. Os primeiros trabalhos identificados sobre a aleatoriedade da série de lucros das empresas são de Little (1962) e Little e Raynor (1967). Ambos pesquisaram as mudanças sucessivas percentuais dos lucros anuais das empresas britânicas como proxy da taxa de crescimento dos lucros e verificaram que não havia estabilidade entre as observações analisadas. O fenômeno foi denominado de Higgledy Piggledy Growth, termo como ficou conhecida a aleatoriedade dessa série. 0 estudo estimulou vários trabalhos envolvendo a aplicação do conceito de caminho aleatório (random walk) em estudos de eficiência de mercado (FAMA, 1965; BROWN, 1993).

Lintner e Glauber (1967) também investigaram o relacionamento entre taxa de crescimento entre períodos sucessivos. Os autores concentraram suas análises em empresas americanas e a taxa de crescimento foi medida como o coeficiente de inclinação nas regressões de variáveis de lucros (apresentadas em logaritmos) em um lapso temporal que compreendia um período de 5 a 10 anos. Constatou-se que havia pouca correlação entre lucros sucessivos. Ball e Watts (1972) chegaram à mesma conclusão ao aplicarem três testes diferentes e acrescentaram que as séries do lucro líquido anual e dos lucros anuais por ação podem ser descritas por um modelo do tipo random walk. Resultados similares foram encontrados por Whittred (1978) e Burgstahler, Caird e Emanuel (1981) ao investigarem o comportamento de lucros de empresas da Austrália e da Nova Zelândia, respectivamente. Foster (1986) ressalta que a constatação de que, na média, os lucros anuais reportados possam ser descritos por um modelo random walk é um dos resultados empíricos mais robustos na literatura acadêmica sobre demonstrações financeiras. 
Brealey (1967) adotou uma metodologia diferente ao investigar a mudança e não a taxa de crescimento em lucros anuais de empresas americanas. Foi verificada a correlação em dados de corte (cross-section) de mudanças de lucros em vários períodos e concluiu-se que as observações seguiam um processo do tipo Martingale $e^{3}$. Reforçando essa constatação, Ball e Brown (1968) utilizaram como premissa que a melhor informação para estimativa do lucro anual é a última observação realizada ${ }^{4}$ e verificaram que esse critério conduz a um erro menor da expectativa do lucro anual das empresas americanas do que seria se fosse usada simplesmente a média das observações passadas ${ }^{5}$.

No que toca a séries de lucros trimestrais, a literatura internacional constatou que os lucros não são totalmente aleatórios em sua natureza. Evidências demonstram que estes seguem um processo auto-regressivo caracterizado por componentes sazonais (FOSTER, 1977; GIFFIN, 1977). O interesse pelos lucros trimestrais é justificado em função das características sazonais de determinadas indústrias e o fato de que o lucro trimestral ser mais freqüente, refletindo de modo mais acurado as necessidades do mercado, que o preferem em relação a previsões de lucros anuais (KOTHARI, 2001)

A propriedade de aleatoriedade dos lucros (random walk) é instigante e desafiadora. Ao contrário da aleatoriedade dos preços das ações, que seria uma decorrência lógica da eficiência de mercado, a teoria econômica não prevê um comportamento de aleatoriedade nos lucros. Os lucros contábeis não representam uma capitalização de fluxos de caixas futuros, tais como os preços das ações. Portanto, não existe uma razão econômica para esperar que os lucros sigam um random walk (WATTS \& ZIMMERMAN, 1990).

\section{METODOLOGIA}

\subsection{DADOS}

Os dados foram obtidos da base de dados Economatica para o período compreendido entre o primeiro trimestre de 1995 e o último trimestre de 2006. Apenas firmas brasileiras participaram da seleção e o IPCA (Índice de Preços ao Consumidor Amplo ${ }^{6}$ ) foi utilizado como deflator da série de dados. Portanto, os dados estão apresentados em valores de dezembro de 2006.

A seleção inicial retornou uma base de 48 observações (48 trimestres) para 436 empresas, ou seja, 20.928 observações ao todo. Um segundo filtro foi aplicado, excluindo empresas financeiras, uma vez que é aceito na literatura acadêmica que tais empresas possuem características próprias do segmento de atuação, que criam obstáculos para fins de comparação com firmas inseridas em outros mercados (FRANCIS et al., 2000; PENMAN \&

\footnotetext{
${ }^{3}$ Seja $Y_{1}, Y_{2} \ldots$ variáveis aleatórias com expectativas. Então a seqüência $\left\{Y_{t}\right\}$ é um processo do tipo Martingale se $E\left(Y_{t+1} \mid Y_{0}, \ldots, Y_{t}\right)=Y_{t}$, para todo t.

${ }^{4}$ Ou seja $E\left(Y_{t}\right)=Y_{t-1}$, onde $Y_{t}$ é o lucro no período $t$.

${ }^{5} \mathrm{O}$ objeto do presente estudo é o lucro trimestral. Contudo, uma primeira percepção do comportamento da série de lucro anual pôde ser constatada pelo teste da função de autocorrelação (não reportado). Esse teste foi aplicado na amostra selecionada, considerando os lucros anuais (somatório dos lucros trimestrais). 0 resultado não permite rejeitar a hipótese de aleatoriedade da série de lucros anuais para o período de 1995 a 2006.

${ }^{6}$ Índice de Preços calculado pelo Instituto Brasileiro de Geografia e Estatística.
} 
SOUGIANNIS, 1998). O terceiro e último filtro objetivou eliminar empresas com dados perdidos ou insuficientes. Como parâmetro, foi adotada a razão de $70 \%$ sobre o período abrangido na seleção. Se, por um lado, tal procedimento pode conduzir ao viés de sobrevivência (ELTON et al., 1996), de outro ele se configura como um procedimento alternativo para o expurgar os outliers (observações extremas) ${ }^{7}$. Esse procedimento foi adotado em decorrência da sensibilidade da análise em relação a poucas observações ou dados perdidos. Conjugados, os dados filtrados correspondem a 10.844 observações da série de Lucro Operacional e 10.841 observações da série de Lucro Líquido.

O efeito do critério de seleção adotado é de superestimar a importância das tendências, uma vez que a amostra contém apenas empresas com dados disponíveis para mais de $70 \%$ da série. Ball e Watts (1972) ensinam que o procedimento de restringir as observações às empresas com dados existentes para a maior parte da série tem como conseqüência concentrar as análises apenas em firmas "sobreviventes" e, assim, os decréscimos observados nas séries de lucros serão poucos e subestimados. A expectativa será, então, de desvios positivos não esperados, remetendo a uma tendência de lucros crescentes, uma vez que a parte relativa às chances de desvios negativos foi removida pelo procedimento de seleção.

O impacto do viés dos desvios positivos não esperados em relação à expectativa genuína de tendência (devido aos reinvestimentos, por exemplo) extrapola o objetivo do presente estudo. A análise incluiria a verificação das razões que explicam as quebras estruturais dentro da série temporal, que poderiam ser distintas em relação ao segmento econômico ${ }^{8}$. A tarefa fica, então, sugerida e reservada a estudos futuros.

Dois tipos de medida de lucros foram utilizados no estudo, sendo eles:

* - Lucro Operacional (LO); e

- - Lucro Líquido (LL).

\subsection{TESTES DE NORMALIDADE}

Muitos métodos de análise de dados (teste $t$, ANOVA, regressão linear etc) dependem da suposição de que os dados foram coletados de uma distribuição Gaussiana. Há diversos métodos para verificar o ajustamento da série de dados à hipótese de distribuição normal, sendo o Jarque-Bera, o Anderson-Darling, o Ryan-Joiner, o Kolmogorov-Smirnov, o Shapiro-Wilk e o critério Cramér-von-Mises alguns dos mais conhecidos. A discussão detalhada de cada um desses testes pode ser encontrada em livros-texto (JUDGE et al., 1988, por exemplo) ou nos artigos de referência. Com o objetivo de verificar o comportamento das séries de dados (em relação à hipótese nula de normalidade), foram aplicados dois desses testes: Anderson-Darling (ANDERSON \& DARLING, 1952) e Ryan-Joiner (RYAN \& JOINER, 1976).

Os dados das séries de lucro - operacional e líquido - foram submetidos aos testes de normalidade selecionados aplicando-se a média das observações trimestrais. Os resultados estão apresentados no Gráfico 1. A baixa probabilidade indicada no $p$-value permite rejeitar, em ambos os testes, a hipótese nula de normalidade das séries de LO e

\footnotetext{
${ }^{7}$ Para maiores detalhes, vide Foster (1986).

${ }^{8}$ Explica-se: cada segmento econômico pode apresentar choques originados por determinantes distintos, tais como fatores macroeconômicos (taxa de juros, variações cambiais etc.) ou microeconômicos (monopólio, por exemplo).
} 
LL. A constatação preliminar de não normalidade das variáveis envolvidas condicionou a escolha por testes não paramétricos para identificação da aleatoriedade das séries analisadas. Uma vez que esses testes não definem previamente um modelo estrutural ${ }^{9}$, sua escolha no estudo é ainda reforçada pelo fato de não ser utilizada nenhuma teoria da firma ou de mensuração do lucro nas análises.

\subsection{TESTES NÃO PARAMÉTRICOS}

O primeiro teste não paramétrico aplicado foi o de "Mudanças Médias", que acompanha as variações do sinal (positivo ou negativo) entre observações consecutivas de uma série de dados. O teste assume mudanças simetricamente distribuídas e considera como indicação de tendência o número de aumentos (diminuições) em relação às diminuições (aumentos) dos valores esperados.

0 quadro 1 demonstra as mudanças médias observadas nos dados trimestrais da série de lucro operacional e de lucro líquido. Os valores comparados representam as médias das observações do trimestre em análise. A coluna "Mudança" do quadro 1 representa a variação observada entre o valor do trimestre em relação ao trimestre imediatamente anterior. Já os acréscimos representam o somatório das variações positivas observadas na série em análise, enquanto que os decréscimos se referem às variações negativas.

Um teste alternativo para tendência é investigar a mudança em médias das empresas (FAMA, 1965), isto é, agregar lucros de empresas e verificar o sinal das mudanças nas séries agregadas é o teste de carreiras. No presente estudo, foram aplicados dois enfoques diferentes. 0 primeiro deles foi o teste de Geary, usado para verificar uma forma específica de independência: a aleatoriedade de um arranjo seqüencial de desvios (observado em forma de sinais) dentro de uma série finita (BALL \& WATTS, 1972). Uma carreira é definida como uma seqüência ininterrupta de sinais positivos ou negativos, tendo seu comprimento igual ao número de elementos. A intuição do teste é a seguinte: se houver carreiras demais, significa que a série muda de sinal com freqüência, indicando correlação serial negativa. Se, por outro lado, houver poucas carreiras, essa situação sugere autocorrelação positiva (GUJARATI, 2000).

Para a hipótese nula de que os sucessivos resultados são independentes, o número de carreiras se distribui normalmente com:

$$
\mu_{R}=\frac{2 N_{1} N_{2}}{N}+1 \quad \text { e } \quad \sigma_{R}^{2}=\frac{2 N_{1} N_{2}\left(2 N_{1} N_{2}-N\right)}{N^{2}(N+1)}
$$

onde $R$ é o número de carreiras, $\mu_{R}$ e $\sigma_{R}^{2}$ são a média e a variância de $R$, respectivamente, $N_{1}$ é o número de sinais positivos, $N_{2}$ é o número de sinais negativos e $N=N_{1}+N_{2}$. Se a série for aleatória, deve-se esperar que o número de carreiras, $R$, se encontre entre $\left\lfloor\mu_{R} \pm 1,96 \sigma_{R}^{2}\right\rfloor$ com $95 \%$ de confiança (GUJARATI, 2000).

\footnotetext{
${ }^{9} \mathrm{O}$ modelo é especificado a partir dos dados.
} 
O segundo teste não é construído observando-se os sinais das variações e sim a média da série. Ele é encontrado em alguns pacotes estatísticos, como o "Minitab". Sua definição de carreira é o conjunto de observações consecutivas em que todas elas não se distanciam em valor superior a média das observações. 0 número observado de carreiras é comparado com o seu número esperado. A probabilidade calculada indica que, dado um nível de significância, existe evidência suficiente para concluir que os dados não são aleatoriamente distribuídos. Os resultados dos dois testes de carreiras estão apresentados no quadro 2.

O teste de carreiras oferece alguma indicação da origem da aleatoriedade (ou não) da série. Um excesso de carreiras reais sobre esperadas sugere um mecanismo de compensação (variações positivas são compensadas por variações negativas), enquanto um déficit é uma evidência da persistência na direção da mudança observada.

Outro teste não-paramétrico para a identificação da aleatoriedade é o das "Diferenças Sucessivas Quadradas Médias". ${ }^{10}$ Para sua execução, a variável $\delta^{2}$ é definida como:

$$
\delta^{2}=\sum_{t T=1}^{N-1}\left(Y_{t-1}^{\prime}-Y_{t}^{\prime}\right)^{2} / 2(N-1)
$$

onde $Y_{t}^{\prime}$ são as diferenças sucessivas, $\delta^{2}$ é a estimativa não viesada da variância de $Y^{\prime}$, se $Y^{\prime}$ for identicamente e independentemente distribuída com variância finita. Uma segunda estimativa da variância é necessária:

$$
s^{2}=\sum_{t=1}^{N}\left(Y_{t}^{\prime}-\bar{Y}^{\prime}\right)^{2} / N
$$

onde $\bar{Y}^{\prime}$ é a diferença média. Então $s^{2}$ é a estimativa usual da variância sob as mesmas premissas. Se as premissas são violadas, então a razão $\delta^{2} / s^{2}$ mede a força e a origem da violação. Se uma tendência de persistência existe, então a razão deverá ser baixa. Por outro lado, se a série flutua com bastante freqüência, então a razão deverá ser alta. Sob a premissa de um processo distribuído independentemente com variância definida, a razão $\varepsilon=\delta^{2} / s^{2}$ tem $\mu_{\varepsilon}=1 \quad$ e $\sigma_{\varepsilon}^{2}=\frac{N-2}{N^{2}-1}$ sendo a distribuição próxima da normal. Por sua vez, a estatística $Z=\frac{1-\varepsilon}{\sigma_{\varepsilon}}$ é normal $(0,1)$.

0 quadro 3 demonstra a estatística $\varepsilon$ e $Z$ das diferenças sucessivas observadas nas séries de lucro operacional e lucro líquido. A interpretação do teste é a seguinte: se a estatística $Z$ for zero, a indicação é de um processo independente. Valores positivos ou negativos, por sua vez, indicam persistência e flutuação, respectivamente.

\footnotetext{
${ }^{10}$ A descrição metodológica deste teste é baseada na discussão promovida por Ball \& Watts (1972).
} 
Como teste final, verificou-se a autocorrelação da série de dados (Gráfico 2). Hanke et al. (2001) ensinam que a autocorrelação é a correlação entre a variável, defasada em um ou mais períodos, com ela mesma. O teste da função de autocorrelação (ACF Autocorrelation Function) é utilizado para determinar esse grau de associação da variação no decorrer do tempo. A função é definida como:

$$
r_{k}=\frac{\sum_{t=1}^{n-k}\left(Y_{t}-\bar{Y}\right)\left(R_{t+k}-\bar{Y}\right)}{\sum_{t=1}^{n}\left(Y_{t}-\bar{Y}\right)^{2}}
$$

onde $r_{k}$ é o coeficiente de autocorrelação, $k$ é o número de defasagens, and $Y_{t}$ representa a variável observada.

Dois elementos importantes na estimativa da autocorrelação são o desvio padrão e a estatística Box Pierce Q (BPQ). O desvio padrão mede o coeficiente de autocorrelação para um intervalo individual e identifica a sua significância, enquanto que a estatística Box Pierce Q mede a significância do coeficiente de aucorrelação no nível agregado.

O desvio padrão $\sigma_{k}$ é definido como $\sqrt{\frac{1+2 \sum_{t=1}^{k-1} \theta_{t}^{2}}{N}}$ onde $\mathrm{N}$ é o número total de observações e $\theta_{k}$ é a autocorrelação na defasagem $(k)$.

Já a estatística Box Pierce Q é identificada como $N(N+2) \sum_{t=1}^{k} \frac{Y_{t}^{2}}{N-t}$.

\section{ANÁLISE DOS RESULTADOS}

Preliminarmente aos resultados auferidos nos testes, cabe ressaltar algumas características identificadas nas séries analisadas. Algumas estatísticas descritivas (média, desvio, mediana, quartis superior e inferior) foram calculadas e se referem aos dados normalizados pelos ativos totais, considerando-se o procedimento de seleção de empresas (detalhado na seção anterior). A motivação adjacente às mudanças observadas nas variáveis de um ano para outro não foram investigadas, seja ela conjuntural ou microeconômica, por ultrapassar o escopo do presente trabalho.

A média foi de 0,0045 e $-0,0338$ para as séries de Lucro Operacional e Lucro Líquido, respectivamente, demonstrando que o resultado não-operacional e itens extraordinários têm um impacto significativo para explicar o afastamento entre aquelas variáveis de LO e LL. Outro fator que influencia nesse afastamento é a heterogeneidade dos dados que, embora reduzida pelo procedimento de normalização das variáveis pelo ativo total, ainda é bastante dispersa em relação à sua média. 0 desvio padrão de 0,2610 do LO e 5,0247 do LL corroboram essa assertiva. A heterogeneidade dos dados ainda pode ser verificada pelos quartis, cujo valor mínimo (máximo) é $-19,0000(1,5000)$ e -102,3600 $(481,7500)$ para LO e LL, respectivamente. Essas variações demonstram que os valores extremos não foram totalmente eliminados com o procedimento de seleção dos dados, que considerou apenas as empresas com mais de 70\% de disponibilidade de observações para o período analisado. Para reduzir esse viés, foi utilizada a mediana, uma vez que tal medida apresenta menor sensibilidade a observações extremas (GREENE, 2003; DECHOW, 1992). 
Assim, nos testes que consideram a média das observações, como é o caso do teste alternativo de carreiras, foi utilizada a média das medianas.

0 teste de mudanças médias (quadro 1) não é um teste robusto de aleatoriedade, mas oferece uma primeira percepção sobre o comportamento das variações. Uma "mudança" é considerada como a diferença entre a observação atual e a imediatamente anterior. Um "acréscimo" ("decréscimos") representa uma variação positiva (negativa) da "mudança”. A intuição é a de que, para grandes amostras, o número de variações não pode ser concentrado em um dos pólos (positivo ou negativo), se os dados forem realmente aleatórios. Os dados por trimestre apresentaram 26 (25) acréscimos e 21 (22) decréscimos para a série de LO (LL), fato que não permitiu rejeitar a hipótese de aleatoriedade para os dados.

Os testes de carreira apresentam-se mais robustos que o teste de mudanças médias. No teste de sinais (teste de Geary) detectou-se que 46,4\% (39,9\%) das séries de LO (LL), por empresa, podiam ser consideradas aleatórias, indicando que a maior parte da amostra selecionada não possuía tal característica. A constatação é corroborada por um teste alternativa de carreira, executado pelo pacote estatístico Minitab. 0 teste alternativo acusou um total de 4 (15) carreiras na série de LO (LL) para um total esperado de 24,62 (25). 0 p-value de 0,0000 $(0,0035)$ permite rejeitar a hipótese nula de aleatoriedade de dados.

O teste das “Diferenças Sucessivas Quadradas Médias” possui algumas das características do teste de carreiras. Os resultados da aplicação desse teste encontram-se descritos no Quadro 3. Conforme já mencionado, se a media de $\varepsilon$ é próxima de 1 ou $Z$ é próximo de zero, tem-se indicação de que o processo é independente. Por outro lado, valores positivos ou negativos de $Z$ indicam persistência ou flutuação, respectivamente. 0 valor médio estimado de $\varepsilon$ para o LO e LL não é próximo o suficiente de 1 (afastamento maior que 2 desvios padrões), fato que permite rejeitar a hipótese de aleatoriedade dos dados. Analogamente, os valores encontrados para a estatística $Z$, são negativos $(-2,11$ e 2,78, para LO e LL, respectivamente), não só indicando que as series não são aleatórias como também sugerindo a presença de autocorrelação negativa.

A autocorrelação na série de dados de lucro operacional lucro trimestral está representada nos correlogramas do Gráfico 2. A Figura A demonstra que as autocorrelações nos primeiras três defasagens são significativas diferentes de zero para a série de LO. A estatística $Q$ calculada para doze defasagens é 19,67 ao passo que a estatística $Q$ calculada foi de 39,57, rejeitando a hipótese de aleatoriedade dos dados da série de LO. Já a Figura $B$ demonstra que há autocorrelação na $1^{\mathrm{a}}, 3^{\mathrm{a}}$ e $4^{\mathrm{a}}$ defasagens para a série de LL. A estatística Q observada foi de 32,52 contra 19,67 para a calculada, considerando um período de 12 defasagens. Assim como foi identificado na série de lucro operacional, as constatações rejeitam a hipótese de aleatoriedade na série de lucro líquido.

\section{CONSIDERAÇÕES FINAIS}

Este artigo analisou as propriedades das séries temporais de empresas brasileiras durante o período de janeiro de 1995 a dezembro de 2006.

A evidência empírica, auferida pelos testes aplicados (mudanças médias, teste de carreiras, diferenças sucessivas e correlação serial), sugere que o processo de geração de lucros trimestrais das empresas brasileiras segue um processo autoregressivo, corroborando com resultados obtidos por Foster (1977), Griffin (1977), Kothari (2001), entre outros, para o mercado norte-americano. 
O fenômeno de autocorrelação negativa verificado dos lucros indica uma reversão a média (mean reversion), entretanto esse fato isolado não asseguraria uma maior previsibilidade dos lucros trimestrais em relação ao random-walk.

Como razões econômicas para a reversão a média dos lucros podem ser citadas: i) a competição no mercado que impede que uma lucratividade acima da média se mantenha no longo prazo, existindo uma inevitável reversão. Nesse ambiente, as firmas com desempenho medíocre tendem a desaparecer, sobrevivendo apenas aquelas que revertem os resultados abaixo da média em direção a média; e ii) o conservadorismo contábil que motiva os gestores a antecipar perdas. O reconhecimento de perdas torna as perdas menos permanentes o que induz a uma autocorrelação negativa. e iii) a sazonalidade em algumas indústrias que torna inevitável a verificação de reversões em torno da média.

Quanto a oportunidades para pesquisas futuras, o campo é fértil. Num primeiro momento, poder-se-ia modelar as séries de lucros trimestrais para objetivos de previsão e avaliação de empresas. Tais modelos poderiam aplicar os mais recentes desenvolvimentos da econometria como os modelos das classes ARCH e GARCH.

Uma segunda motivação seria verificar se os resultados encontrados por Hong e Stein (1999), nos EUA, sobre a previsibilidade dos retornos das ações e seu relacionamento com variações de lucros se aplica ao mercado brasileiro.

Finalmente, apesar da consistência dos resultados, as constatações referem-se apenas à amostra examinada. Eventuais extrapolações para outras séries de dados envolvendo lucros (abrangência temporal ou forma de mensuração diferenciada, por exemplo) devem ser efetuadas com cautela.

\section{REFERÊNCIAS}

ANDERSON, T. W.; DARLING, D. A. Asymptotic theory of certain "goodness-of-fit" criteria based on stochastic processes. Annals of Mathematical Statistics, 23, 1952.

BALL, R.; BROWN, P. Portfolio Theory and Accounting. Journal of Accounting Research, v. VII, 2, 1969.

; WATTS, R. Some Time Series Properties of Accounting Income. The Journal of Finance, v. 27, 3, 1972.

BREALEY, R. A. Statistical Properties of Successive Changes in Earnings. Keystone Custodian Funds, 967.

BROWN, L. Earnings Forecasting Research: its implications for capital market research, International Journal of Forecasting, v. 9, 1993.

BURGSTAHLER, D., DICHEV, I. Earnings management to avoid earnings decreases and losses. Journal of Accounting and Economics, 24, 1, p. 99-126, 1997.

CAIRD, K. G.; EMANUEL, D. M. Some Time-Series Properties of Accounting Income Numbers. Australian Journal of Management, Dec., 1-341981.

COPELAND, R. M. Income Smoothing. Journal of Accounting Research, suplemento do v. IV, 1968.

DECHOW, P. M. Accounting Earnings and Cash Flow as Measures of Firm Peformance: The Role of Accounting Accruals. New York. University of Rochester. New York, 1992. 
M.; RICHARDSON, S. A.; TUNA, I. Why Are Earnings Kinky? An Examination of the Earnings Management Explanation. Review of Accounting Studies, 8, 2-3, p. 355-384, 2003.

DONNELLY, R. Earnings Persistence, Losses and the Estimation of Earnings Response Coefficients. Abacus, v. 38, 1, 2002.

ELTON, E. J.; GRUBER, M. J.; BLAKE, C. R. Survivorship Bias and Mutual Fund Performance, The Review of Financial Studies, v. 9, 4, 1996.

FAMA, E. The Behavior of Stock-Market Prices. The Journal of Business, v. 38, 1, 1965.

FERNÁNDEZ, P. Valuation Using Multiples: How do Analysts Reach their Conclusions? [S.l.]: Social Science Research Network, 2001. Disponível em: <http://papers.ssrn.com>. Acesso em 19 out. 2003.

FIPECAFI. Manual de Contabilidade das Sociedades por Ações (aplicável às Demais Sociedades), 6 ed. São Paulo: Atlas, 2003.

FRANCIS, J.; OLSSON, P.; OSWALD, D. Comparing the Accuracy and Explainability of Dividend, Free Cash Flow, and Abnormal Earnings Equity Value Estimates. Journal of Accounting Research, v. 38,spring, p. 45-70, 2000.

FOSTER, G. Financial Statement Analysis, 2.ed.. Englewood Cliffs: Prentice Hall, 1986.

FOSTER, G, Quartely accounting data: time-series properties and predictive-ability results. Accounting Review, 52, 1-21, 1977.

GAGNON, J.-M. Purchase Versus Pooling of. Interests: The Search for a Predictor. Journal of Accounting Research, suplemento do v. IV, 1967.

GRIFFIN, P. The time-series behavior of quarterly earnings: preliminary evidence. Journal of Accounting Research 15, 71-83, 1977.

GREENE, W. Econometric Analysis. New Jersey: Prentice Hall, 5. ed. 2003.

GORDON, M. J. Postulates, Principles and Research in Accounting. Accounting Review, v. XXXIX, 2, 1964.

HAMMAN, W. D.; JORDAAN, A.C.; SMIT, E.V.D. Earnings Changes: a Random Walk? Same South African Evidence. The Investment Analysts Journal, 41, p. 5, 2002.

HEPWORTH, S. R. Smoothing Periodic Income. Accounting Review, v. XXVIII, 1, 1953.

HONG, H., STEIN, J., A unified theory of underreaction, momentum trading and overreaction in asset markets, Journal of Finance, 54, p. 2.143-2.184, 1999.

IBGE - Instituto Brasileiro de Geografia e Estatística. Índice de Preços ao Consumidor Amplo. Disponível em << http://www. ibge.gov.br >>. Acesso em 03 jan. 2007.

KOTHARI, S. P., Capital Markets Research in Accounting, Journal of Accounting and Economics, p. 105-231, 2001.

JUDGE, G. G.; CARTER, R. H.; WILLIAM, E. G.; HELMUT, L.; TSOUNG-CHAO L. Theory and Practice of Econometrics, 2. ed. NY: John Wiley \& Sons, 1988.

LINTNER, J.; GLAUBER, R. Higgledy Piggledy Growth in America. Modern Developments in Investment Management, 2 ed., Hindale IL: Dryden Press, 1967.

LITTLE, L. D. Higgledy Piggledy Growth. Bulletin of the Oxford Institute of Economics and Statistics, v. 24,4, p. 387-412, 1962.

LITTLE, I. M. D.; RAYNER, A. C. Higgledy Piggledy Growth again. Economica. New Series, v. 34,135, p. 326-329, 1967. 
OHLSON, J. A. Earnings, Book Values, and Dividends in Equity Valuation. Contemporary Accounting Research, v. 11, p. 661-687, 1995.

PATON, W.; LITTLETON, A. An Introduction to Corporate Accounting Standards. Sarasota: American Accounting Association, 1940.

PENMAN, S. H.; SOUGIANNIS, T. A Comparison of Dividend, Cash Flow, and Earnings Approaches to Equity Valuation. Contemporary Accounting Research, v. 15, 3, fall, p. 343-383, 1998.

RAYNER, A.C., LITTLE, L.D. Higgledy Piggledy Growth Again. Oxford: Basil Blackwell, 1966.

SMITH, C.; WARNER, J. On Financial Contracting: An Analysis of Bond Covenants. Journal of Financial Economics, v. 6, p. 117-161, 1979.

GUJARATI, D. N. Econometria Básica. São Paulo: Makron Books, 2000.

HANKE, J. E.; REITSCH, A. G.; WICHERN, D. W. Business Forecasting, 7.ed. New Jersey: Prentice Hall, 2001.

HAYN, C. The Information Content of Losses. Journal of Accounting and Economics, v. 20, 2, September, p. 125-153, 1995..

RYAN, T.A.; JOINER, B.L. Normal Probability Plots and Tests for Normality Technical Report, Statistics Department, The Pennsylvania State University, 1976.

STRICKLAND, S. G. Discretionary Accounting Changes in Relation to Income Smoothing, Firm Characteristics and Bankruptcy Prediction Models. Texas A\&M University, 1981.

WATTS, R ; ZIMMERMAN, J, Positive Accounting Theory: a ten-year perspective, The Accounting Review. 65, p. 131-156, 1990.

WHITTRED, G. P. The Time-Series Behaviour of Corporate Earnings. Australian Journal of Management, oct., p. 195-202, 1978.

\section{ENDEREÇO DOS AUTORES}

Universidade Federal da Bahia

Praça da Piedade, 06

Salvador, BA - Brasil

40070-010

Centro Sócio-Econômico

Departamento de Ciências Econômicas Universidade Federal de Santa Catarina Caixa-Postal: 476 - Trindade

Florianópolis, SC - Brasil

88049970
Superintendência Regional do Estado de Santa Catarina Departamento de Polícia Federal

Setor Técnico Científico

Av. Gov. Irineu Bornhausen, 4744 - Centro

Florianópolis, SC - Brasil

88025-201

Milanez \& Coelho Consultoria Empresarial

Rua dos Cravos, 210 - Daniela

Florianopolis, SC - Brasil

88053-030 


\section{Gráfico 1 - Testes de Normalidade}

O gráfico apresenta os testes de normalidade aplicados nas séries de lucros operacional e líquido - para os trimestres compreendidos entre janeiro de 1995 e dezembro de 2006. Todas as empresas brasileiras listadas na Bolsa de Valores de São Paulo - BOVESPA - foram selecionadas, excluindo-se as financeiras. A mediana das observações trimestrais foi utilizada nos testes de normalidade, que compreenderam as abordagens Anderson-Darling e Ryan-Joyner. Em ambas, a constatação da baixa probabilidade $-p$ value - permite rejeitar a hipótese nula de ajustamento das séries à distribuição normal. A Figura A apresenta o teste de Anderson-Darling e a Figura B refere-se ao teste de RyanJoyner.
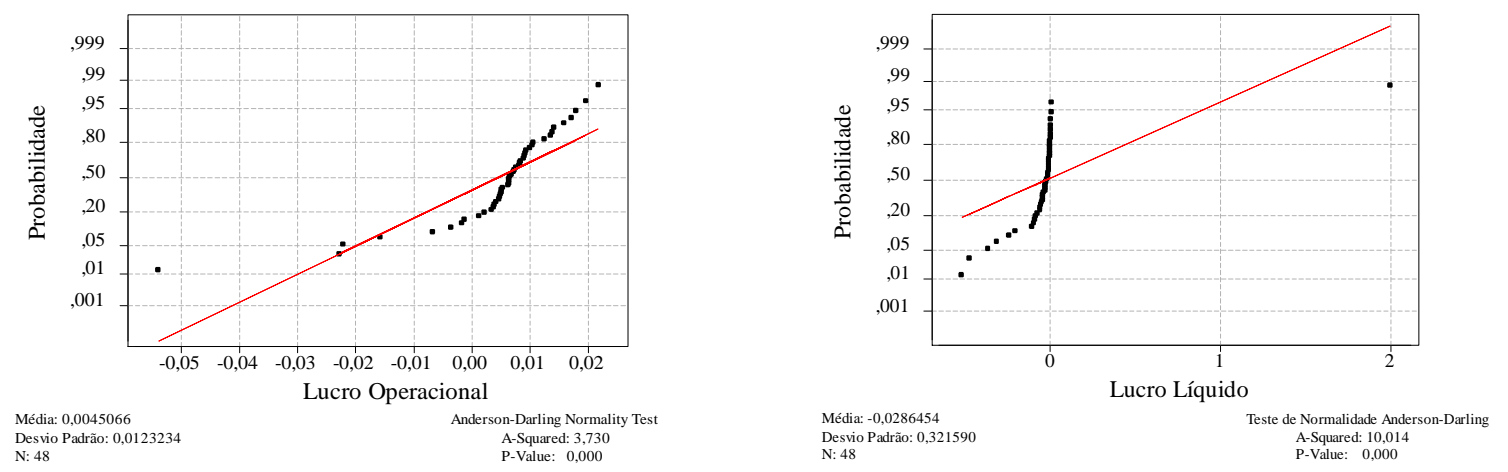

Figura A: Teste Anderson-Darling

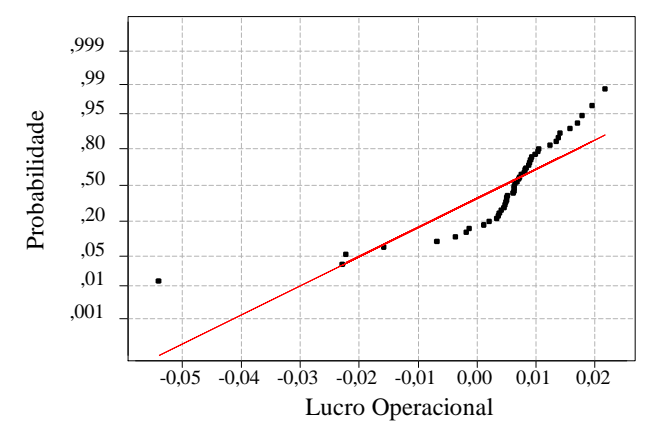

Média: 0,0045066
Desvio Padrão: 0,0123234
N: 48

Lucro Operacional
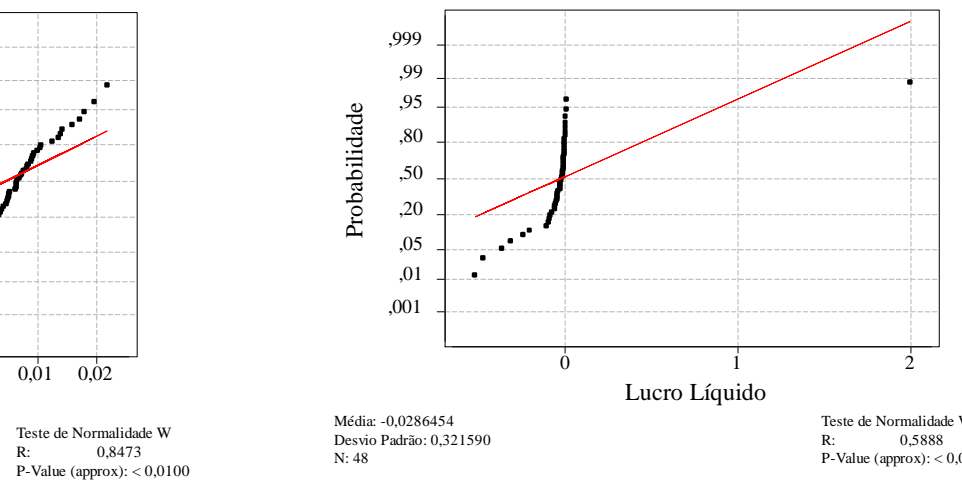

Média: $-0,0286454$

Dessio Padrão: 0,321590
N: 48

Teste de Normalidade W
R: $\quad 0,5888$
P-Value (approx): $<0,0100$

Figura B: Teste Ryan-Joyner 


\section{Quadro 1 - Mudanças Médias}

O quadro apresenta as mudanças médias verificadas nas séries de lucros operacional e líquido - para os trimestres compreendidos entre janeiro de 1995 e dezembro de 2006. Todas as empresas brasileiras listadas na Bolsa de Valores de São Paulo - BOVESPA - foram selecionadas, excluindo-se as financeiras. A média das medianas das observações trimestrais foi utilizada no cálculo das variações. A coluna "Mudança" corresponde à diferença observada entre o valor atual e o imediatamente anterior. Para as duas séries, o número de acréscimos e decréscimos observados não permite rejeitar a hipótese de aleatoriedade dos dados.

\begin{tabular}{|c|c|c|c|c|c|c|c|c|c|}
\hline \multirow{2}{*}{ Período } & \multicolumn{2}{|c|}{ Lucro Operacional } & \multicolumn{2}{|c|}{ Lucro Líquido } & \multirow{2}{*}{ Período } & \multicolumn{2}{|c|}{ Lucro Operacional } & \multicolumn{2}{|c|}{$\underline{\text { Lucro Líquido }}$} \\
\hline & Valor & Mudança & Valor & Muda & & Valor & Mudança & Valor & Iança \\
\hline $1^{\circ}$ Trim 1995 & 0,00526 & & 0,00707 & & $1^{\circ}$ Trim 2001 & 0,0122 & 0,0 & 0,00014 & -0, \\
\hline $2^{\circ}$ Trim 1995 & 0,00488 & $-0,00038$ & 0,00758 & 0,0005 & $2^{\circ}$ Trim 2001 & 0,01332 & 0,00112 & 0,0019 & 0,00176 \\
\hline $3^{\circ}$ Trim 1995 & 0,0004 & $-0,00448$ & 0,00447 & $-0,00311$ & $3^{\circ}$ Trim 2001 & 0,01233 & $-0,00099$ & 0,00254 & 0,00063 \\
\hline $4^{\circ}$ Trim 1995 & $-0,00211$ & $-0,00251$ & $-0,00061$ & $-0,00508$ & $4^{\circ}$ Trim 2001 & 0,01634 & 0,00402 & 0,01049 & 0,00796 \\
\hline $1^{\circ}$ Trim 1996 & 0,00149 & 0,0036 & 0,00149 & 0,0021 & $1^{\circ}$ Trim 2002 & 0,01358 & $-0,00276$ & 0,0017 & $-0,00879$ \\
\hline $2^{\circ}$ Trim 1996 & 0,00351 & 0,00202 & 0,00453 & 0,00304 & $2^{\circ}$ Trim 2002 & 0,01285 & $-0,00073$ & $-0,00452$ & $-0,00622$ \\
\hline $3^{\circ}$ Trim 1996 & 0,00656 & 0,00305 & 0,00547 & 0,00094 & $3^{\circ}$ Trim 2002 & 0,01664 & 0,00379 & $-0,00706$ & $-0,00254$ \\
\hline $4^{\circ}$ Trim 1996 & 0,00757 & 0,00101 & $-0,00049$ & $-0,00596$ & $4^{\circ}$ Trim 2002 & 0,02007 & 0,00344 & 0,01244 & 0,01951 \\
\hline $1^{\circ}$ Trim 1997 & 0,00496 & $-0,00261$ & 0,00311 & 0,0036 & $1^{\circ}$ Trim 2003 & 0,0161 & $-0,00398$ & 0,00575 & $-0,00669$ \\
\hline $2^{\circ}$ Trim 1997 & 0,00644 & 0,00148 & 0,00519 & 0,00209 & $2^{\circ}$ Trim 2003 & 0,01426 & $-0,00184$ & 0,00645 & 0,0007 \\
\hline $3^{\circ}$ Trim 1997 & 0,00891 & 0,00247 & 0,00732 & 0,00213 & $3^{\circ}$ Trim 2003 & 0,01833 & 0,00408 & 0,0043 & $-0,00215$ \\
\hline $4^{\circ}$ Trim 1997 & 0,00644 & $-0,00248$ & 0,00248 & $-0,00485$ & $4^{\circ}$ Trim 2003 & 0,02113 & 0,0028 & 0,00833 & 0,00402 \\
\hline $1^{\circ}$ Trim 1998 & 0,00294 & $-0,0035$ & 0,00261 & 0,00013 & $1^{\circ}$ Trim 2004 & 0,01915 & $-0,00198$ & 0,00499 & $-0,00333$ \\
\hline $2^{\circ}$ Trim 1998 & 0,00618 & 0,00324 & 0,00675 & 0,00414 & $2^{\circ}$ Trim 2004 & 0,01972 & 0,00057 & 0,0065 & 0,00151 \\
\hline $3^{\circ}$ Trim 1998 & 0,00693 & 0,00076 & 0,00428 & $-0,00246$ & $3^{\circ}$ Trim 2004 & 0,02281 & 0,00308 & 0,01415 & 0,00765 \\
\hline $4^{\circ}$ Trim 1998 & 0,00362 & $-0,00331$ & 0,00128 & $-0,003$ & $4^{\circ}$ Trim 2004 & 0,02525 & 0,00244 & 0,01315 & $-0,001$ \\
\hline $1^{\circ}$ Trim 1999 & 0,01015 & 0,00653 & $-0,00803$ & $-0,00931$ & $1^{\circ}$ Trim 2005 & 0,01876 & $-0,00649$ & 0,00648 & $-0,00667$ \\
\hline $2^{\circ}$ Trim 1999 & 0,00965 & $-0,00051$ & 0,00116 & 0,0092 & $2^{\circ}$ Trim 2005 & 0,01537 & $-0,00339$ & 0,01032 & 0,00384 \\
\hline 3 Trim 1999 & 0,01318 & 0,00353 & 0,00075 & $-0,00041$ & $3^{\circ}$ Trim 2005 & 0,01743 & 0,00206 & 0,00693 & $-0,00339$ \\
\hline $4^{\circ}$ Trim 1999 & 0,00678 & $-0,0064$ & 0,00845 & 0,00769 & $4^{\circ}$ Trim 2005 & 0,01637 & $-0,00106$ & 0,01015 & 0,00322 \\
\hline $1^{\circ}$ Trim 2000 & 0,00823 & 0,00145 & 0,00381 & $-0,00464$ & $1^{\circ}$ Trim 2006 & 0,01467 & $-0,0017$ & 0,006 & $-0,00415$ \\
\hline $2^{\circ}$ Trim 2000 & 0,01107 & 0,00284 & 0,00455 & 0,00074 & $2^{\circ}$ Trim 2006 & 0,01384 & $-0,00083$ & 0,00694 & 0,00094 \\
\hline $3^{\circ}$ Trim 2000 & 0,01265 & 0,00158 & 0,00401 & $-0,00054$ & $3^{\circ}$ Trim 2006 & 0,01833 & 0,00449 & 0,00772 & 0,00078 \\
\hline $4^{\circ}$ Trim 2000 & 0,01179 & $-0,00085$ & 0,00297 & $-0,00104$ & $4^{\circ}$ Trim 2006 & 0,01871 & 0,00038 & 0,01481 & 0,00709 \\
\hline
\end{tabular}

Total de Acréscimos: 26 (Lucro Operacional) e 21 (Lucro Líquido)

Total de Decréscimos: 25 (Lucro Operacional) e 22 (Lucro Líquido) 


\section{Quadro 2 - Testes de Carreira}

O quadro apresenta os testes de carreiras aplicados nas séries de lucros operacional e líquido - para os trimestres compreendidos entre janeiro de 1995 a dezembro de 2006. Todas as empresas brasileiras listadas na Bolsa de Valores de São Paulo - BOVESPA - foram selecionadas, excluindo-se as financeiras. A média das observações trimestrais foi utilizada nos testes, que compreenderam dois enfoques alternativos. Em ambos, a constatação da baixa probabilidade - $p$-value - permite rejeitar a hipótese nula de aleatoriedade da série de dados. O Painel A apresenta o teste de Geary e o Painel B refere-se ao teste alternativo.

Painel A: Teste de Geary

\begin{tabular}{ccc}
\hline & $\begin{array}{c}\text { Lucro } \\
\text { Operacional }\end{array}$ & Lucro Líquido \\
\cline { 2 - 3 }$N_{1}$ & 22 & 21 \\
$N_{2}$ & 20 & 21 \\
$N$ & 42 & 42 \\
$R$ & 25 & 26 \\
Média & 21,95 & 22,00 \\
Variância & 10,20 & 10,24 \\
Desvio Padrão & 3,19 & 3,20 \\
Limite Inferior (LI) & 18,76 & 18,80 \\
Limite Superior (LS) & 25,15 & 25,20 \\
Abaixo LI & 19 & 19 \\
Acima LS & 129 & 147 \\
Séries Aleatórias & 128 & 110 \\
\% Aleatórias & $46,4 \%$ & $39,9 \%$ \\
\hline
\end{tabular}

Painel B: Teste Alternativo

\begin{tabular}{lrr}
\hline & \multicolumn{1}{c}{$\begin{array}{c}\text { Lucro } \\
\text { Operacional }\end{array}$} & Lucro Líquido \\
\cline { 2 - 3 } Média (K) & 0,0118 & 0,0048 \\
$N^{0}$ Observado de & 4 & 15 \\
Carreiras & & \\
$N^{0}$ Esperado de & 24,625 & 25 \\
Carreiras & & \\
Observações Acima & 27 & 24 \\
Observações Abaixo & 21 & 24 \\
P-Value & 0,00 & 0,0035 \\
\hline
\end{tabular}




\section{Quadro 3 - Diferenças Sucessivas Quadradas Médias}

O quadro apresenta os testes das Diferenças Sucessivas e Quadradas Médias aplicados nas séries de lucros - operacional e líquido - para os trimestres compreendidos entre janeiro de 1995 e dezembro de 2006. Todas as empresas brasileiras listadas na Bolsa de Valores de São Paulo - BOVESPA - foram selecionadas, excluindo-se as financeiras. 0 Painel A corresponde ao teste aplicado na série de Lucro Operacional e o Painel $B$, na série de Lucro Líquido.

Painel A: Diferenças Sucessivas Médias Quadradas do Lucro Operacional

\begin{tabular}{|c|c|c|c|c|c|}
\hline & $\bar{Y}^{\prime}$ & N & $\delta^{2}$ & $s^{2}$ & $\varepsilon$ \\
\hline Média das Séries & $(0,00)$ & 38,279 & 0,137 & 0,214 & 1,375 \\
\hline Desvio Padrão & 0,00 & 5,717 & 3,80 & 9,234 & 0,032 \\
\hline \multicolumn{2}{|l|}{$\varepsilon$ Média Esperada } & 1 & \multicolumn{2}{|c|}{$\begin{array}{l}\varepsilon-\varepsilon \text { Esperado } \\
\text { Afastamento de } \varepsilon \text { em } n^{\circ} \text { de }\end{array}$} & 0,375 \\
\hline \multicolumn{2}{|c|}{$\varepsilon$ Variância Esperada } & 0,025 & \multicolumn{2}{|c|}{$D P$} & 2,380 \\
\hline \multicolumn{2}{|c|}{$\varepsilon$ Desvio Padrão (DP) Esperado } & 0,157 & \multicolumn{2}{|l|}{$z$} & $(2,11)$ \\
\hline
\end{tabular}

Painel B: Diferenças Sucessivas Médias Quadradas do Lucro Líquido

\begin{tabular}{|c|c|c|c|c|c|}
\hline & $\bar{Y}^{\prime}$ & N & $\delta^{2}$ & $s^{2}$ & $\varepsilon$ \\
\hline Média das Séries & $(0,00)$ & 38,290 & 46,00 & 69,826 & 1,421 \\
\hline Desvio Padrão & 0,00 & 5,725 & $5,14 \mathrm{E}+05$ & $1,21 \mathrm{E}+06$ & 0,023 \\
\hline \multicolumn{2}{|l|}{$\varepsilon$ Média Esperada } & 1 & \multicolumn{2}{|c|}{$\begin{array}{l}\varepsilon-\varepsilon \text { Esperado } \\
\text { Afastamento de } \varepsilon \text { em } n^{\circ} \text { de }\end{array}$} & 0,421 \\
\hline \multicolumn{2}{|c|}{$\varepsilon$ Variância Esperada } & 0,025 & \multicolumn{2}{|l|}{$D P$} & 2,674 \\
\hline \multicolumn{2}{|c|}{$\varepsilon$ Desvio Padrão (DP) Esperado } & 0,157 & Z & & $(2,78)$ \\
\hline
\end{tabular}




\section{Gráfico 2 - Teste da Função de Autocorrelação}

0 gráfico apresenta os testes da Função de Autocorrelação aplicados nas séries de variações lucros - operacional e líquido - para os trimestres compreendidos entre janeiro de 1995 a dezembro de 2006. Todas as empresas brasileiras listadas na Bolsa de Valores de São Paulo - BOVESPA - foram selecionadas, excluindo-se as financeiras. A mediana das observações trimestrais foi utilizada nos testes. Ficou evidenciada que a estatística Q é maior que o valor Qui-quadrado de 19,6751, considerando um período de 12 defasagens e uma significância de 0,05 . Ou seja, os dados são autocorrelacionados e esse autocorrelação foi verificada no Lucro Operacional na primeira, segunda e terceira defasagens e no Lucro Líquido na primeira, terceira e quarta defasagens.

Figura A: Teste ACF para série de Lucros Operacionais

ACF Lucro Operacional

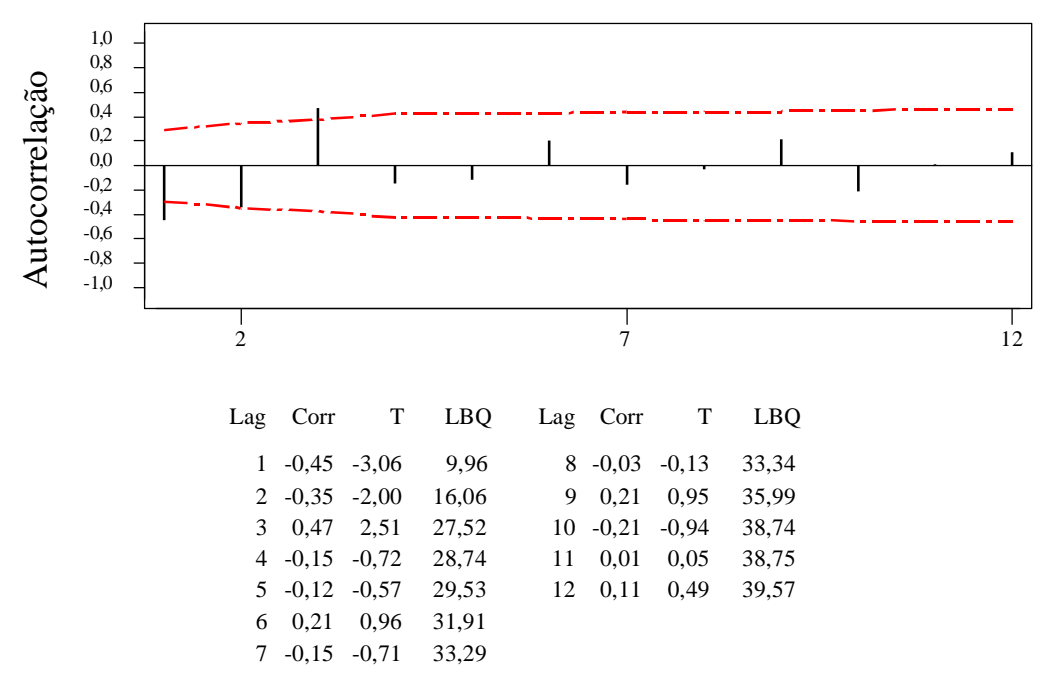

Figura B: Teste ACF para série de Lucro Líquido

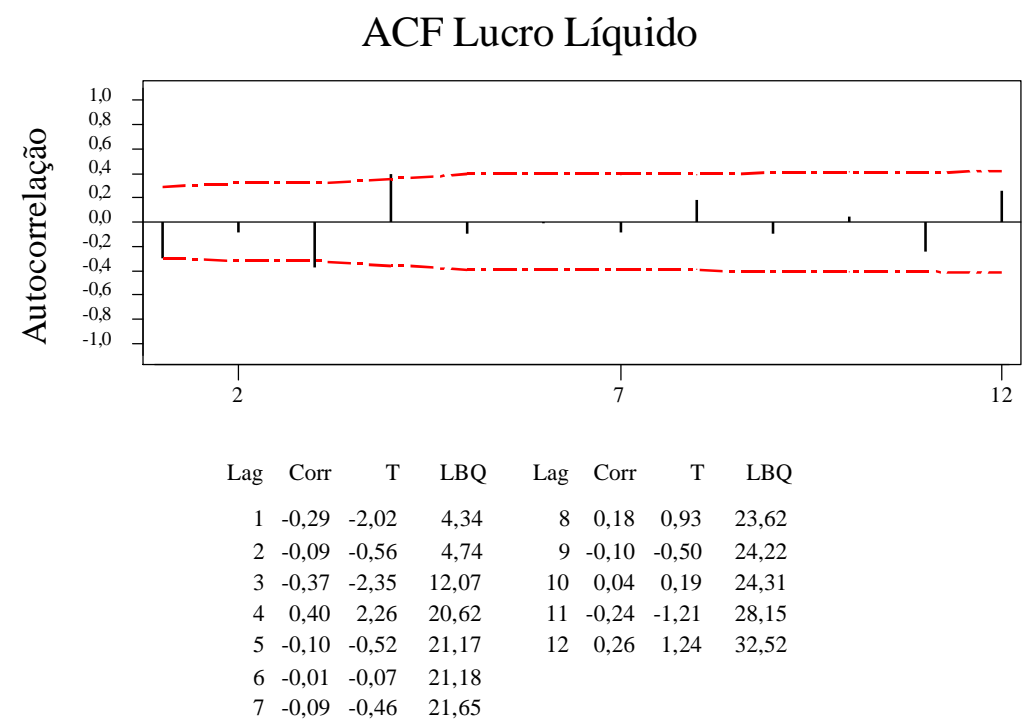

